\title{
No-scale supergravity inflation: A bridge between string theory and particle physics?
}

\author{
John Ellis \\ Theoretical Particle Physics and Cosmology Group, \\ Department of Physics, King's College London, Strand, \\ London WC2R 2LS, United Kingdom \\ Theoretical Physics Department, \\ CERN, CH 1211 Geneva 23, Switzerland \\ John.Ellis@cern.ch
}

\begin{abstract}
The plethora of recent and forthcoming data on the cosmic microwave background (CMB) data are stimulating a new wave of inflationary model-building. Naturalness suggests that the appropriate framework for models of inflation is supersymmetry. This should be combined with gravity in a supergravity theory, whose specific no-scale version has much to commend it, e.g. its derivation from string theory and the flat directions in its effective potential. Simple no-scale supergravity models yield predictions similar to those of the Starobinsky $R+R^{2}$ model, though some string-motivated versions make alternative predictions. Data are beginning to provide interesting constraints on the rate of inflaton decay into Standard Model particles. In parallel, LHC and other data provide significant constraints on no-scale supergravity models, which suggest that some sparticles might have masses close to present experimental limits.
\end{abstract}

Keywords: Inflation; supersymmetry; supergravity; CMB; LHC.

\section{Cosmological Inflation}

For almost two decades now, the cosmological paradigm has been the $\Lambda \mathrm{CDM}$ model, based on the general theory of relativity with the addition of a cosmological constant $\Lambda$. Einstein supposedly regarded the introduction in 1917 of $\Lambda$ as "the biggest blunder of his life," ${ }^{1}$ but nowadays we would beg to differ. Its presence is a possibility allowed by the general principles of relativity, and hence is probably compulsory. The present-day cosmological constant has depressing implications for astronomers in the far future, who will only be able to study our local gravitationally-bound group of galaxies.

However, according to the hypothesis of cosmological inflation ${ }^{2}$, the energy density of the very early universe may have been dominated by an analogous cosmological (almost) constant term that would have generated an epoch of (near-) exponential expansion that could explain why our universe today is such a special solution of Einstein's equations, with size $\gg$ the Planck length and age $\gg$ the Planck time. As an added bonus, quantum fluctuations in this primordial $\Lambda$ could have been the origin of the structures seen in the universe today.

The most important evidence for cosmological inflation, and constraints on models, come from observations of fluctuations in the cosmic microwave background (CMB) radiation, e.g. by the Planck satellite ${ }^{3}$. Together with other cosmological and astrophysical data, these CMB measurements are consistent with the cosmological concordance model in which the total density is very close to the critical density 
$\Omega_{\text {Tot }}=1$ in a flat universe, with the following fractions provided by a present-day cosmological constant and by matter: $\Omega_{\Lambda} \simeq 0.69, \Omega_{\text {matter }} \simeq 0.31$, with most of the latter being cold dark matter $(\mathrm{CDM})^{4}$.

In this talk, I first review aspects of the inflationary paradigm and how it is constrained by CMB data, then discuss models, with emphasis on those based on supersymmetry and (no-scale) supergravity ${ }^{5}$, which may provide a bridge between string theory and the particle physics accessible at colliders ${ }^{6}$.

\section{Slow-Roll Inflationary Models}

Most interpretations of the CMB data adopt the framework of slow-roll inflationary models ${ }^{2}$, in which the near-exponential expansion of the universe would have been driven by the almost constant field energy $V$ of some inflaton scalar field $\phi$ :

$$
\epsilon \equiv \frac{1}{2} M_{P}^{2}\left(\frac{V^{\prime}}{V}\right)^{2}, \quad \eta \equiv M_{P}^{2}\left(\frac{V^{\prime \prime}}{V}\right) \ll 1 .
$$

Quantum fluctuations in $\phi$ would have led to scalar and tensor perturbations in the CMB, and the principal observables are the power-law tilt in the scalar spectrum $n_{s}$, and the tensor-to-scalar ratio $r$, which are given in the slow-roll approximation by

$$
n_{s}=1-6 \epsilon+2 \eta, \quad r=16 \epsilon,
$$

respectively. It is a generic prediction of slow-roll models that the CMB fluctuations should be Gaussian to a very good approximation.

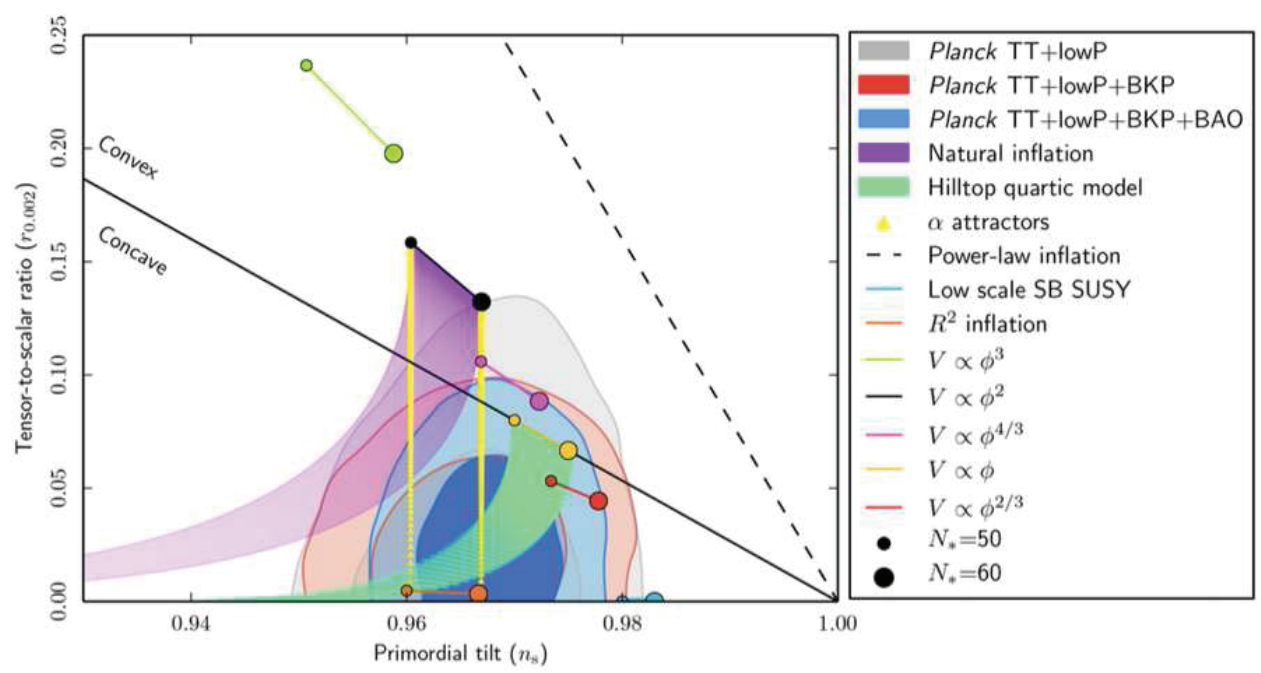

Fig. 1. The marginalized joint $68 \%$ and $95 \%$ CL regions for the tilt in the scalar perturbation spectrum, $n_{s}$, and the relative magnitude of the tensor perturbations, $r$, obtained from the Planck 2015 data and their combinations with BICEP2/Keck Array and/or BAO data ${ }^{3}$, confronted with the predictions of some inflationary models. 
There was great excitement when the BICEP2 experiment reported ${ }^{7}$ the measurement of B-mode polarization in the $\mathrm{CMB}$, which might have been generated by primordial tensor (quantum gravitational-wave) perturbations. However, these are now thought to be consistent with pollution by dust ${ }^{8}$, and a recent combined analysis of BICEP2/Keck array and Planck data yields only an upper limit on $r<0.1^{9}$, as seen in Fig. 1, where we also see that $n_{s} \sim 0.97$.

We also see in Fig. 1 that monomial single-field potentials $\propto \phi^{n}$ are disfavored by the data, whereas the original model of Starobinsky with an $R+R^{2}$ gravitational action $^{10}$ is highly compatible with the data, as would be inflation driven by the Higgs field ${ }^{11}$ (with a nonminimal coupling to gravity) or in no-scale supergravity models ${ }^{5}$, as discussed later. We also note that the experimental restriction on $n_{s}$, in particular, starts to provide an interesting constraint on the number of e-folds of inflationary expansion, $N_{*}$, which is sensitive to how the inflaton decayed into conventional matter ${ }^{12}$, as also discussed later.

\section{Challenges for Inflationary Models}

It is already a challenge to write down a simple model of inflation that is compatible with the CMB data, with simple power-law potentials being excluded as seen in Fig. 1, but there are other, deeper challenges.

How can one link inflation to 'low-energy' physics at colliders? Within the Standard Model of particle physics, the only candidate for the inflaton is the Higgs boson $^{11}$, but naive extrapolation of Standard Model measurements to high energies indicates that the Higgs potential probably turns negative ${ }^{13}$, which is unsuitable for inflation.

Can one link inflation to some other well-motivated extension of the Standard Model? For example, could the inflaton be a supersymmetric partner of a righthanded (singlet) neutrino field ${ }^{14}$, or some sort of axion ${ }^{15}$ ?

At the high end of the energy scale, can one link inflation to Planck-scale physics? Specifically, are there plausible inflatons in string theory, or in compactified string models? These issues are discussed later in this talk.

\section{The Starobinsky Model}

Let us first review the Starobinsky model ${ }^{10}$, which is seen in Fig. 1 to be highly compatible with the $\mathrm{CMB}$ data. It is based on a nonminimal action for general relativity:

$$
S=\frac{1}{2} \int d^{4} x \sqrt{-g}\left(R+\frac{R^{2}}{6 M^{2}}\right),
$$

where $M$ is some mass parameter that is unknown a priori. It would seem superficially that the action (3) does not contain a scalar inflaton field. However, when one makes a conformal transformation to the Einstein frame ${ }^{16}$ (in which the dependence on $R$ is purely linear) one find a scalar field model with a characteristic 
potential:

$$
S=\frac{1}{2} \int d^{4} x \sqrt{\tilde{g}}\left[\tilde{R}+\left(\partial_{\mu} \phi\right)^{2}-\frac{3}{2} M^{2}\left(1-e^{-\sqrt{\frac{2}{3}} \phi}\right)^{2}\right] .
$$

Scalar CMB perturbations were first calculated in this model in Ref. 17, and their small magnitude requires $M \sim 10^{13} \mathrm{GeV} \ll M_{P}$, i.e. a coefficient of the $R^{2}$ term in (3) that is $\gg 1$ in natural units. The value of the scalar tilt $n_{s}$ and the magnitude of the tensor-to-scalar ratio $r \sim 3 \times 10^{-3}$ are highly compatible with the available CMB data, as already seen in Fig. 1.

\section{Higgs Inflation}

William of Occam might have suggested using the (only established) Higgs scalar $h$ as the inflaton, which would require a nonminimal coupling to gravity ${ }^{11}$ :

$$
S=\frac{1}{2} \int d^{4} x \sqrt{-g}\left[-\left(1+\xi h^{2}\right) R+\left(\partial_{\mu} h\right)^{2}-\frac{\lambda}{2}\left(h^{2}-v^{2}\right)^{2}\right],
$$

where $\xi$ is an unknown parameter that must be $\gg 1, \lambda$ is the quartic Higgs selfcoupling and $v$ is the Higgs vev. When $1 \ll \xi \ll 10^{17}$, upon transforming to the Einstein frame one finds

$$
S=\frac{1}{2} \int d^{4} x \sqrt{-\tilde{g}}\left[\tilde{R}+\left(\partial_{\mu} \chi\right)^{2}-\frac{\lambda}{2 \xi^{2}}\left(1+e^{-\sqrt{\frac{2}{3}} \chi}\right)^{-2}\right] .
$$

The resulting effective potential gives successful inflation when $\chi \gg 1$, where the potential is similar to that of the Starobinsky model (though clearly different at small $\chi)$.

The required value of $\xi \sim 15,000$, and one may ask how natural that is. However, a more serious practical problem is that one needs $\lambda>0$ for field values beyond the Planck scale, whereas the best available calculations indicate that probably $\lambda<0$ because of renormalization by the top quark ${ }^{13}$. One accurate calculation indicates that $\lambda$ turns negative at a scale $\Lambda$ :

$$
\begin{aligned}
\log \frac{\Lambda}{\mathrm{GeV}}= & 11.3+1.0\left(\frac{M_{h}}{\mathrm{GeV}}-125.66\right)-1.2\left(\frac{m_{t}}{\mathrm{GeV}}-173.10\right) \\
& +0.4\left(\frac{\alpha_{3}\left(M_{Z}\right)-0.1184}{0.0007}\right) .
\end{aligned}
$$

Inserting the current experimental values of the Higgs mass $M_{h}=125.09 \pm$ $0.24 \mathrm{GeV}^{18}$, the top mass $m_{t}=173.34 \pm 0.27 \pm 0.71 \mathrm{GeV}^{19}$ and the stronginteraction coupling $\alpha_{3}\left(M_{Z}\right)=0.1177 \pm 0.0013^{20}$, one estimates that the Higgs self-coupling $\lambda$ turns negative at $\ln (\Lambda / \mathrm{GeV})=10.0 \pm 1.0$.

Negative $\lambda$ leads to metastability of the electroweak vacuum and the problem that most of the early universe would have been sucked into the region where the potential is negative ${ }^{21}$. Physics beyond the Standard Model is needed to avoid these issues, and supersymmetry is one of the possibilities. 


\section{Inflation Cries Out for Supersymmetry and (No-Scale) Supergravity}

There are many other motivations for supersymmetry, and inflation is one of them ${ }^{22}$. Models of inflation involve a scalar inflaton field that is elementary, at least at energies $\ll M_{P}$, and obtaining the right magnitude of perturbations requires a mass $\ll M_{P}$ (typically $\sim 10^{13} \mathrm{GeV}$ ) and/or a small inflaton self-coupling $\lambda \ll 1$. Both of these are technically natural in supersymmetric theories.

The first supersymmetric models of inflation were based on global supersymme$\operatorname{try}^{22}$. However, experience (general relativity, the Standard Model) teaches us that the only good symmetry is a local symmetry, and the same should apply to supersymmetry. The local version of supersymmetry necessarily involves local spacetime coordinate transformations, and is supergravity ${ }^{23}$. Clearly also, any discussion of early universe cosmology needs gravity, and supergravity is the way to combine gravity and supersymmetry.

However, there is an issue with generic supergravity models when matter is included, which is that their effective potentials typically have 'holes' with depths $\sim \mathcal{O}\left(M_{P}^{2}\right)$. These make any treatment of cosmology problematic, let alone inflation. There is, however, an exception, namely the class of no-scale supergravity models ${ }^{24}$. The effective potentials in this class of supergravity models have flat directions, and the scalar potential resembles that in globally supersymmetric models, with controlled corrections, making it possible to construct interesting models of particle physics $^{25}$.

An added motivation for considering no-scale supergravity models is that they arise naturally in compactifications of string theory ${ }^{26}$. Therefore, no-scale models of inflation may offer some prospects for making contact with some underlying variant of string theory.

\section{No-Scale Supergravity Models of Inflation}

The first no-scale supergravity models of inflation appeared in the $1980 \mathrm{~s}^{27,28}$, largely motivated by the 'unholy' form of the effective potential. This approach was revived in $2013^{5}$ in response to the new, high-precision CMB data from Planck. Supergravity models are characterized by a Hermitian Kähler potential $K$ that specifies the internal geometry of the scalar fields $\phi$, whose kinetic terms are $\left(\partial^{2} K / \partial \phi^{i} \partial \phi_{j}^{*}\right) \partial_{\mu} \phi^{i} \partial^{\mu} \phi_{j}^{*}$. The simplest no-scale inflationary model contains two complex scalar fields:

$$
K=-3 \ln \left(T+T^{*}-\frac{|\phi|^{2}}{3}\right),
$$

and the potential interactions between the scalar fields are characterized by a superpotential $W$, which we assumed to have the form

$$
W=\frac{\mu}{2} \phi^{2}-\frac{\lambda}{3} \phi^{3},
$$


as first studied by Wess and Zumino ${ }^{29}$. We assumed that the field $T$, which could be interpreted as a modulus of string compactification, is fixed by string dynamics: $T=c / 2$, in which case the effective Lagrangian for the inflaton field $\phi$ is

$$
\mathcal{L}_{\text {eff }}=\frac{c}{\left(c-\frac{|\phi|^{2}}{3}\right)^{2}}\left|\partial_{\mu} \phi\right|^{2}-\frac{\hat{V}}{\left(c-\frac{|\phi|^{2}}{3}\right)^{2}}: \hat{V}=\left|\frac{\partial W}{\partial \phi}\right|^{2} .
$$

We note that the prefactors in the Lagrangian terms in (10) modify the form of the effective potential that one would have obtained in the globally-supersymmetric case, when one transforms to a canonically-normalized inflaton field.

The Wess-Zumino superpotential gives good inflation already in the global case $^{30}$, and we found ${ }^{5}$ this to be possible also in its no-scale supergravity incarnation for $\lambda / \mu \sim 1 / 3$, as seen in Fig. 2. Indeed, for the specific case $\lambda=\mu / 3$, shown as the black line in Fig. 2, the effective scalar potential is identical with that in the original Starobinsky model $(3,4)$.

Looking beyond the Starobinsky limit, one can consider generic potentials of the form $^{31}$

$$
V=A\left(1-\delta e^{-B \phi}+\mathcal{O}\left(e^{-2 B \phi}\right)\right) .
$$

In such models, the most prominent inflationary predictions are

$$
n_{s}=1-2 B^{2} \delta e^{B \phi_{*}}, \quad r=8 B^{2} \delta^{2} e^{-2 B \phi_{*}}, \quad N_{*}=\frac{1}{B^{2} \delta} e^{B \phi_{*}},
$$

where $\Phi_{*}$ is the value of the inflaton field that yields $N_{*}$ e-folds of inflation. It is clear from (12) that $n_{s}$ is in a one-to-one relationship with $N_{*}$, and that $r$ may be

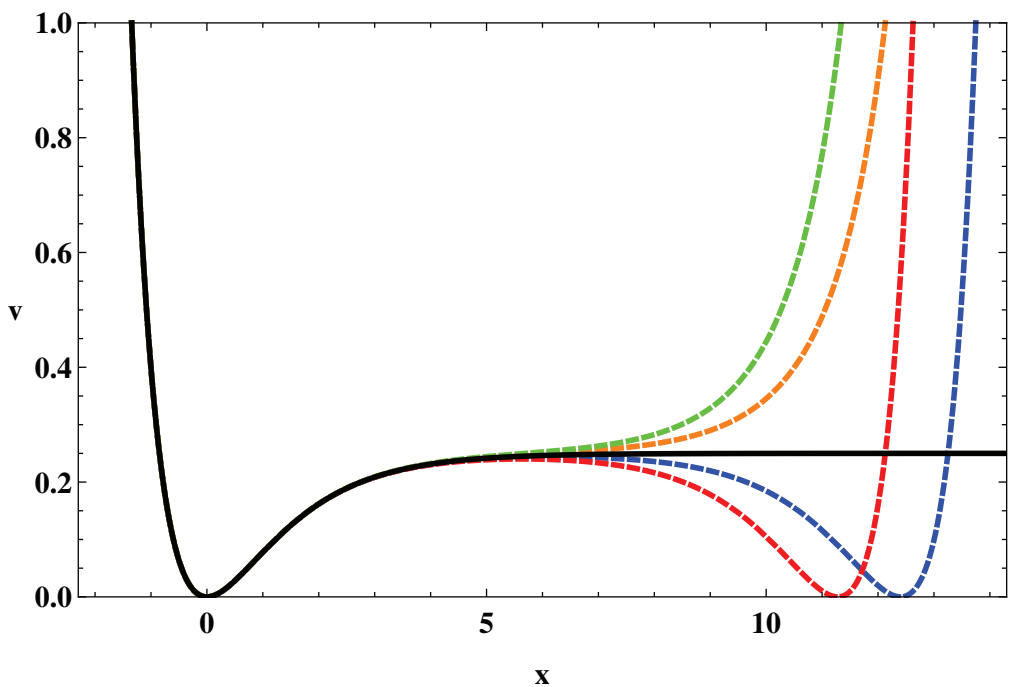

Fig. 2. The inflationary potential $V$ in the Starobinsky $R+R^{2}$ model $^{10}$ (solid black line) compared with its form in various no-scale models ${ }^{5}$ (dashed colored lines). 
adjusted, for any fixed value of $N_{*}$, by varying the coefficient $B$ in the exponential in $(11) .^{\mathrm{a}}$

As an example how this freedom could be exploited, one can consider models in which the no-scale property is shared among several moduli fields ${ }^{31}$ :

$$
K \ni-\sum_{i} \ln \left(T_{i}+T_{i}^{*}\right): N_{i}>0, \quad \sum_{i} N_{i}=3,
$$

as is characteristic of generic string compactifications. In this case, if the inflaton is identified with $T_{i}$ one has

$$
B=\sqrt{\left(\frac{2}{N_{i}}\right)}, \quad r=\frac{4 N_{i}}{N_{*}^{2}},
$$

with the specific choice of a single modulus with $N_{i}=3$ corresponding to the Starobinsky model $(3,4)$. A measurement of $r$ could therefore, within this no-scale supergravity framework, provide a unique window on the phenomenology of string compactification.

\section{A No-Scale Inflationary Model to Fit Them All}

It is possible within the no-scale supergravity framework also to construct models of inflation that allow larger tensor/scalar values. For example, if one considers ${ }^{33}$ a string-motivated modified no-scale Kähler potential of the form

$$
K=-3 \ln (T+\bar{T})+\frac{|\phi|^{2}}{(T+\bar{T})^{2}},
$$

and superpotential

$$
W=\sqrt{\frac{3}{4}} \frac{m}{a} \phi(T-a),
$$

one can identify the inflaton with a combination of components $\rho, \sigma$ of the modulus field $T$ :

$$
T=-a\left(e^{-\sqrt{\frac{2}{3}} \rho}+i \sqrt{\frac{2}{3}} \sigma\right),
$$

with the effective Lagrangian (when $\phi=0$ ):

$$
\mathcal{L}=\frac{1}{2} \partial_{\mu} \rho \partial^{\mu} \rho+\frac{1}{2} e^{2 \sqrt{\frac{2}{3}} \rho} \partial_{\mu} \sigma \partial^{\mu} \sigma-\frac{3}{4} m^{2}\left(1-e^{-\sqrt{\frac{2}{3}} \rho}\right)^{2}-\frac{1}{2} m^{2} \sigma^{2} .
$$

This clearly reduces to a Starobinsky-like no-scale model along the $\rho$-axis and a quadratic model along the $\sigma$-axis, as seen in Fig. 3 , and the predictions of the model interpolate between the predictions of these limiting cases.

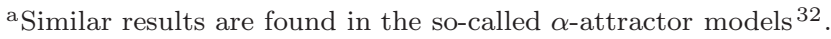




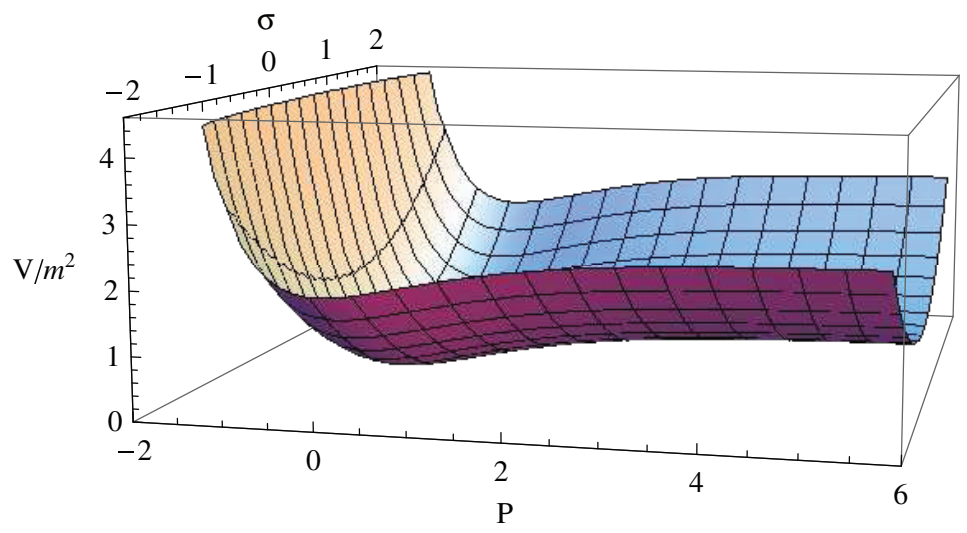

Fig. 3. The potential of the modified no-scale inflationary model $(15,16,17,18)$ in the $(\rho, \sigma)$ plane for $\phi=0^{33}$.

\section{How Many e-Folds of Inflation, and How Does the Inflaton Decay?}

The discussion above shows that the number of e-folds, $N_{*}$, is a key to the phenomenology of inflationary models, and we also see in Fig. 1 that the CMB data are beginning to provide interesting constraints on $N_{*}{ }^{12}$. This can be related to the potential $V_{*}$ at $\phi_{*}$ corresponding to the scale $k_{*}$ :

$$
N_{*}=67-\ln \left(\frac{k_{*}}{a_{0} H_{0}}\right)+\frac{1}{4} \ln \left(\frac{V_{*}^{2}}{M_{P}^{2} \rho_{\mathrm{end}}}\right)+\frac{1-3 w_{\mathrm{int}}}{12\left(1+w_{\mathrm{int}}\right)} \ln \left(\frac{\rho_{\mathrm{reh}}}{\rho_{\mathrm{end}}}\right)-\frac{1}{12} \ln g_{\mathrm{th}},
$$

where $\rho_{\text {end,reh }}$ are the densities at the end of inflation and after reheating, respectively, $w_{\text {int }}$ is the average equation-of-state parameter during inflaton decay and reheating, and $g_{\mathrm{th}}$ is the number of degrees of freedom after reheating ${ }^{2}$.

The inflaton may decay into two-body final states ${ }^{12}$, e.g. in sneutrino inflation:

$$
\Gamma_{\phi}=m \frac{|\lambda|^{2}}{8 \pi},
$$

or into three-body final states such as $H t \bar{t}$ :

$$
\Gamma_{\phi} \sim \frac{m^{3}\left|y_{t}\right|^{2}}{12(8 \pi)^{3} M_{P}^{2}} .
$$

There may also be decays into pairs of vector bosons:

$$
\Gamma_{\phi}=\frac{d^{2}}{32 \pi} m^{3},
$$

where the parameter $d$ also controls the gaugino mass:

$$
m_{1 / 2}=\mathcal{O}\left(\frac{d}{6}\right) m_{3 / 2},
$$

providing a possible direct connection between inflaton decay and sparticle masses. 
How do the CMB data constrain models of inflaton decay ${ }^{12}$ ? The value of $N_{*}$ and hence $r$, in particular, is sensitive to the inflaton decay rate $\Gamma_{\phi}$, since the ratio $\rho_{\text {reh }} / \rho_{\text {end }} \propto \Gamma_{\phi}^{2}$. Thus, a measurement of $r$ can constrain models of inflaton decay. This point is illustrated in Fig. 4 for the Starobinsky-like no-scale supergravity models of inflation whose potentials are shown in Fig. 2. In particular, for the special case $\lambda / \mu=1 / 3$ that reproduces exactly the Starobinsky model, we see that $N_{*} \geq 44$ at the $95 \% \mathrm{CL}$ and $\geq 50$ at the $68 \% \mathrm{CL}$. If the dominant inflaton decay is via a trilinear superpotential coupling $\lambda$ into two-body final states, the inflaton decay rate $\Gamma_{\phi}=|\lambda|^{2} / 8 \pi m_{\phi}$, and these constraints on $N_{*}$ correspond to the constraints $\lambda>10^{-16}$ at the $95 \%$ CL and $>10^{-7}$ at the $68 \% \mathrm{CL}^{12}$.

These bounds are to be compared with those due to upper limits on $\lambda$ from the production in inflaton decays of gravitinos with mass $m_{3 / 2}{ }^{12}$. We find

$$
|\lambda| \lesssim 1.5 \times 10^{-6} \text { for } m_{3 / 2}=6 \mathrm{TeV}
$$

from requiring that long-lived gravitinos do not mess up the success of standard big-bang nucleosynthesis (BBN) calculations, weakening for larger $m_{3 / 2}$ and disappearing altogether if the gravitino is sufficiently heavy to decay before BBN. There is also a constraint

$$
|\lambda|<2.7 \times 10^{-5} \times\left(\frac{100 \mathrm{GeV}}{m_{\mathrm{LSP}}}\right),
$$

- $95 \% \cdot 68 \%$ Planck+BKP+BAO

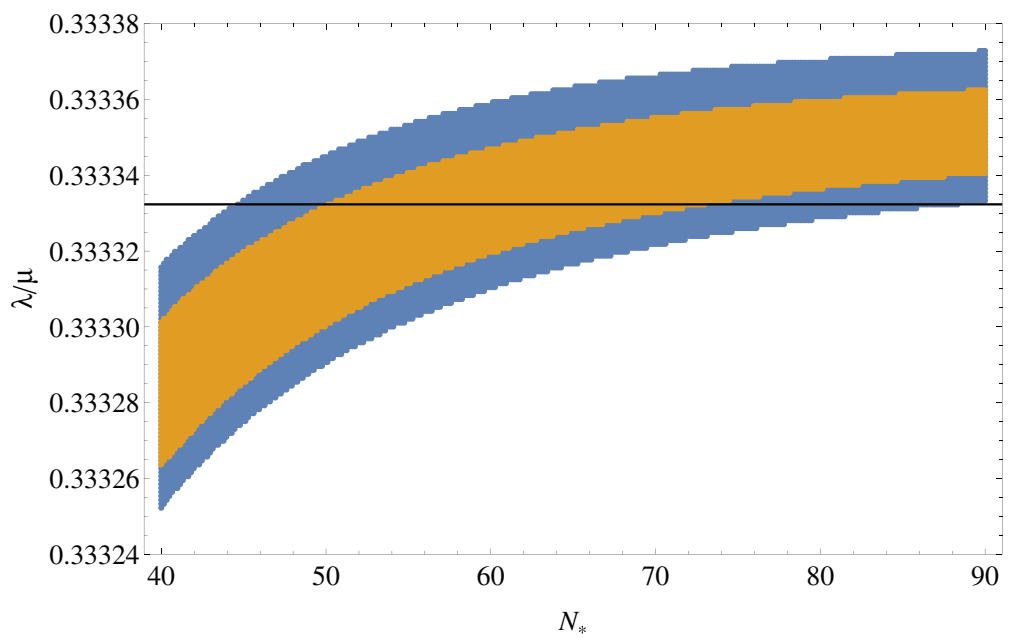

Fig. 4. (Color online) The $68 \%$ and $95 \% \mathrm{CL}$ regions (yellow and blue, respectively) in the $\left(N_{*}, \lambda / \mu\right)$ plane for the no-scale inflationary model ${ }^{5}$ with a matter inflaton field and the WessZumino superpotential ${ }^{12}$. The horizontal black line is for $\lambda / \mu=1 / 3$, the value that reproduces the inflationary predictions of the Starobinsky model ${ }^{5}$. 
from the upper limit on the contribution of the lightest supersymmetric particle (LSP) to the cosmological CDM. The latter constraint, in particular, suggests that $|\lambda| \lesssim 10^{-5}$ for LSP masses in the range of a few hundred $\mathrm{GeV}$.

The constraint on the inflaton decay coupling $y$ from the CMB measurement of $r$ is not yet competitive with the other constraints $(24,25)$, but this can be expected to change in the future.

\section{No-Scale Framework for Particle Physics and Dark Matter}

As seen in Fig. 5, it is possible to construct a no-scale model for particle physics ${ }^{34}$ that incorporates the lower limits on sparticle masses from the LHC, the measured value of the Higgs mass, the constraints from flavor physics and the appropriate cosmological density of supersymmetric dark matter, as well as Starobinsky-like inflation, leptogenesis, small neutrino masses, etc. In much of the model parameter

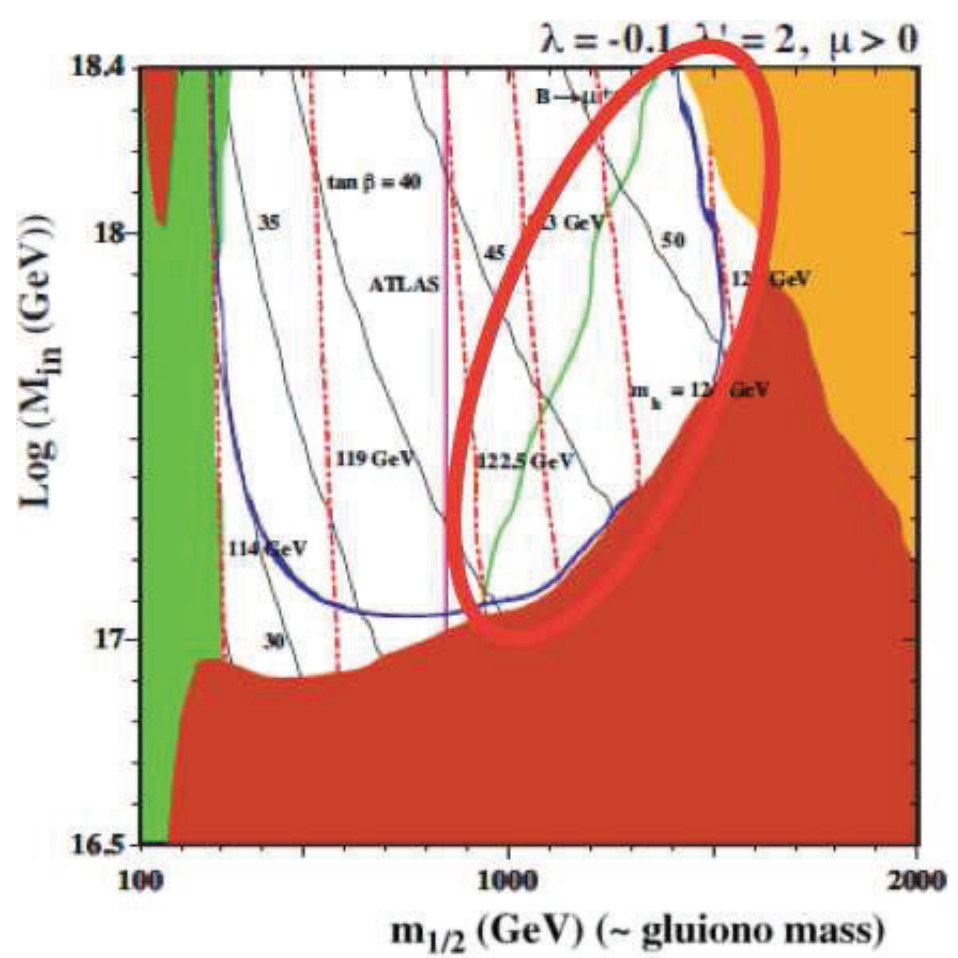

Fig. 5. (Color online) A sample parameter plane in a no-scale model with an SU(5) GUT symmetry (details can be found in Ref. 34). The brown regions are excluded because the LSP is charged, the green regions are excluded by $b \rightarrow s \gamma$, the renormalization-group equations are unstable in the ochre region. The continuous (purple) line is the ATLAS 95\% CL limit on supersymmetric particles, the dash-dotted (red) lines are contours of $m_{H}$, and the solid (green) line marks the $95 \%$ CL upper limit on $\operatorname{BR}\left(B_{s} \rightarrow \mu^{+} \mu^{-}\right)$. The continuous (black) lines are contours of the ratio of supersymmetric Higgs vevs, $\tan \beta$. The relic CDM density lies within the cosmological range along the (dark blue) strip, and the portion inside the red ellipse is compatible with all constraints. 
space compatible with all these constraints, the gluino mass may lie within a factor $\sim 2$ of the lower limit established during Run 1 of the LHC. This (and possibly other supersymmetric particles) may be within reach of future LHC runs, but there are no guarantees!

\section{Summary}

Inflation may solve some of the biggest problems in cosmology, including its age, size, (near-)homogeneity, (near-)flatness, etc., as well as providing the initial seeds for all the structures in the universe. The CMB and other experiments are providing plenty of cosmological data to chew on, and it is now open season for building inflationary models. One promising example is the $R+R^{2}$ model proposed originally by Starobinsky ${ }^{10}$, but the challenge is to relate inflationary models to an underlying fundamental theory such as string, on the one hand, and particle physics, on the other hand. My point of view is that inflation cries out for supersymmetry and that, in the cosmological context, this requires supergravity. From the point of view of string theory as well as to avoid unacceptable 'holes' in the effective potential, it is natural to consider no-scale supergravity. No-scale supergravity readily accommodates models of inflation ${ }^{5}$ with predictions similar to that of the Starobinsky model, though other scenarios are also possible ${ }^{33}$. The CMB data are beginning to constrain the number of e-folds during inflation and hence models of inflaton decay $^{12}$. In turn, these constrain the connection of inflationary models to particle physics at accessible energies ${ }^{34}$. Will the LHC discover supersymmetry? We will have to wait and see!

\section{Acknowledgments}

The author was supported partly by the London Centre for Terauniverse Studies (LCTS), using funding from the European Research Council via the Advanced Investigator Grant 26732, and partly by the STFC Grant ST/L000326/1. He thanks Marcos García, Dimitri Nanopoulos and Keith Olive for collaboration on the topics discussed here.

\section{References}

1. G. Weinstein, arXiv:1310.1033 [physics.hist-ph].

2. J. Martin, C. Ringeval and V. Vennin, Phys. Dark Univ. 5-6 (2014) 75, arXiv:1303.3787 [astro-ph.CO].

3. Planck Collab. (P. A. R. Ade et al.), arXiv:1502.02114 [astro-ph.CO].

4. Particle Data Group Collab. (K. A. Olive et al.), Chin. Phys. C 38 (2014) 090001, doi:10.1088/1674-1137/38/9/090001.

5. J. Ellis, D. V. Nanopoulos and K. A. Olive, Phys. Rev. Lett. 111 (2013) 111301; Phys. Rev. Lett. 111 (2013) 129902, arXiv:1305.1247 [hep-th].

6. J. Ellis, M. A. G. Garcia, D. V. Nanopoulos and K. A. Olive, Class. Quantum Grav. 33 (2016) 094001, doi:10.1088/0264-9381/33/9/094001, arXiv:1507.02308 [hep-ph]. 
7. BICEP2 Collab. (P. A. R. Ade et al.), Phys. Rev. Lett. 112 (2014) 24, 241101, doi:10.1103/PhysRevLett.112.241101, arXiv:1403.3985 [astro-ph.CO].

8. Planck Collab. (R. Adam et al.), Astron. Astrophys. 586 (2016) A133, doi:10.1051/0004-6361/201425034, arXiv:1409.5738 [astro-ph.CO].

9. BICEP2 and Keck Array Collab. (P. A. R. Ade et al.), arXiv:1510.09217 [astro-ph.CO].

10. A. A. Starobinsky, Phys. Lett. B 91 (1980) 99.

11. F. L. Bezrukov and M. Shaposhnikov, Phys. Lett. B 659 (2008) 703, arXiv:0710.3755 [hep-th].

12. J. Ellis, M. A. G. Garcia, D. V. Nanopoulos and K. A. Olive, J. Cosmol. Astropart. Phys. 1507 (2015) 07, 050, doi:10.1088/1475-7516/2015/07/050, arXiv:1505.06986 [hep-ph].

13. D. Buttazzo, G. Degrassi, P. P. Giardino, G. F. Giudice, F. Sala, A. Salvio and A. Strumia, J. High Energy Phys. 1312 (2013) 089, arXiv:1307.3536 [hep-ph].

14. H. Murayama, H. Suzuki, T. Yanagida and J. Yokoyama, Phys. Rev. Lett. 70 (1993) 1912.

15. F. C. Adams, J. R. Bond, K. Freese, J. A. Frieman and A. V. Olinto, Phys. Rev. D 47 (1993) 426 [hep-ph/9207245].

16. K. S. Stelle, Gen. Relativ. Gravit. 9 (1978) 353; B. Whitt, Phys. Lett. B 145 (1984) 176.

17. V. F. Mukhanov and G. V. Chibisov, JETP Lett. 33 (1981) 532.

18. ATLAS and CMS Collab. (G. Aad et al.), Phys. Rev. Lett. 114 (2015) 191803, doi:10.1103/PhysRevLett.114.191803, arXiv:1503.07589 [hep-ex].

19. ATLAS, CDF, CMS and D0 Collab., arXiv:1403.4427 [hep-ex].

20. D. d'Enterria and P. Z. Skands (eds.), arXiv:1512.05194 [hep-ph].

21. M. Fairbairn and R. Hogan, Phys. Rev. Lett. 112 (2014) 201801, doi:10.1103/PhysRevLett.112.201801, arXiv:1403.6786 [hep-ph]; A. Hook, J. Kearney, B. Shakya and K. M. Zurek, J. High Energy Phys. 1501 (2015) 061, doi:10.1007/JHEP01(2015)061, arXiv:1404.5953 [hep-ph].

22. J. R. Ellis, D. V. Nanopoulos, K. A. Olive and K. Tamvakis, Phys. Lett. B 118 (1982) 335; R. Holman, P. Ramond and G. G. Ross, Phys. Lett. B 137 (1984) 343.

23. D. Z. Freedman, P. van Nieuwenhuizen and S. Ferrara, Phys. Rev. D 13 (1976) 3214, doi:10.1103/PhysRevD.13.3214; S. Deser and B. Zumino, Phys. Lett. B 62 (1976) 335, doi:10.1016/0370-2693(76)90089-7.

24. E. Cremmer, S. Ferrara, C. Kounnas and D. V. Nanopoulos, Phys. Lett. B 133 (1983) 61.

25. J. R. Ellis, A. B. Lahanas, D. V. Nanopoulos and K. Tamvakis, Phys. Lett. B 134 (1984) 429, doi:10.1016/0370-2693(84)91378-9.

26. E. Witten, Phys. Lett. B 155 (1985) 151.

27. A. S. Goncharov and A. D. Linde, Class. Quantum Grav. 1 (1984) L75.

28. J. R. Ellis, K. Enqvist, D. V. Nanopoulos, K. A. Olive and M. Srednicki, Phys. Lett. B 152 (1985) 175, Erratum-ibid. 156B (1985) 452.

29. J. Wess and B. Zumino, Phys. Lett. B 49 (1974) 52, doi:10.1016/0370-2693(74) 90578-4.

30. D. Croon, J. Ellis and N. E. Mavromatos, Phys. Lett. B 724 (2013) 165, arXiv: 1303.6253 [astro-ph.CO].

31. J. Ellis, D. V. Nanopoulos and K. A. Olive, J. Cosmol. Astropart. Phys. 1310 (2013) 009, arXiv:1307.3537. 
32. R. Kallosh and A. Linde, J. Cosmol. Astropart. Phys. 1307 (2013) 002, doi:10.1088/ 1475-7516/2013/07/002, arXiv:1306.5220 [hep-th].

33. J. Ellis, M. A. G. Garcia, D. V. Nanopoulos and K. A. Olive, J. Cosmol. Astropart. Phys. 1408 (2014) 044, doi:10.1088/1475-7516/2014/08/044, arXiv:1405.0271 [hep$\mathrm{ph}]$.

34. J. Ellis, D. V. Nanopoulos and K. A. Olive, Phys. Rev. D 89 (2014) 043502, doi:10.1103/PhysRevD.89.043502, arXiv:1310.4770 [hep-ph]. 